\title{
Estudio de habilidades metacognitivas en docentes universitarios colombianos
}

\author{
Study of Metacognitive Awareness \\ in Colombian University Teachers
}

Andrea Barrera-Bernal* y José Bayona-Umbarila**

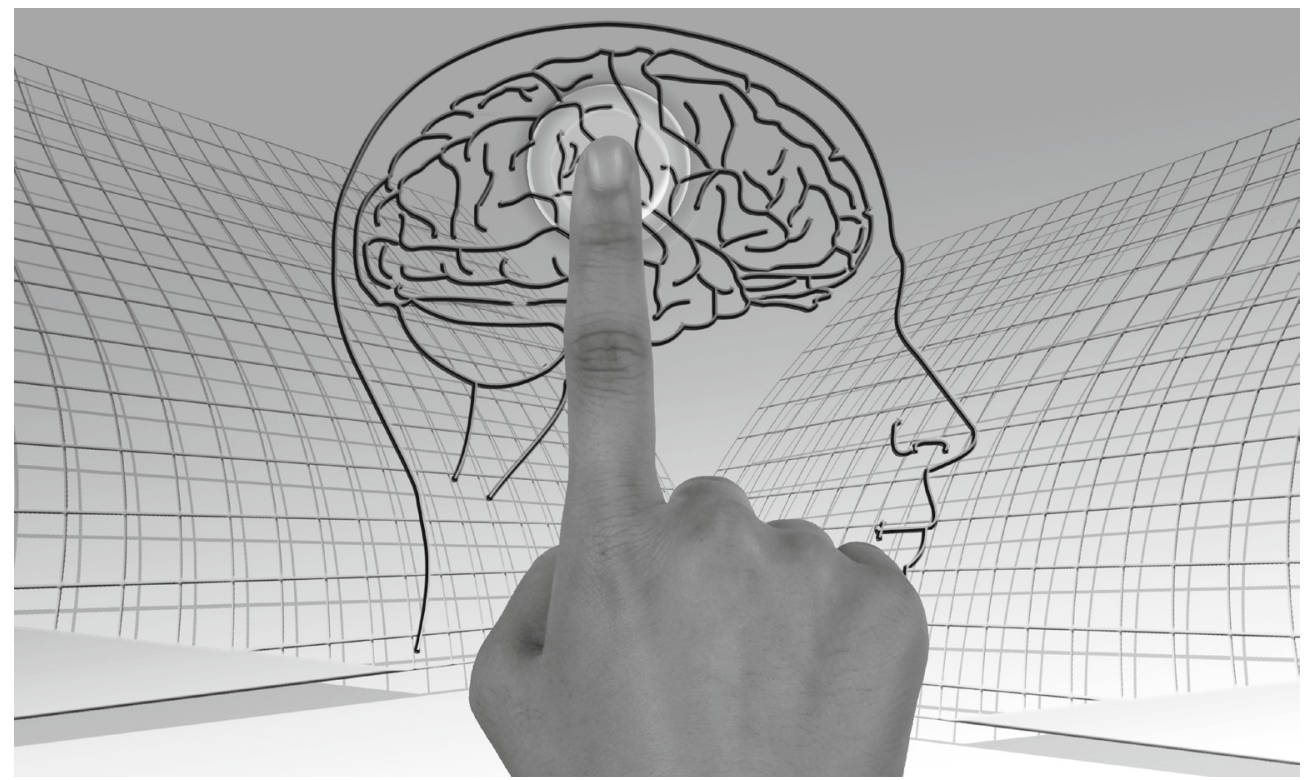

\section{Resumen}

Se realiza un estudio sobre habilidades metacognitivas que reportan 118 docentes universitarios de Bogotá a través de un inventario de habilidades metacognitivas para docentes (MAIT). A partir de los datos recolectados se realiza una validación del

Citar este artículo como: Barrera-Bernal, A. y Bayona-Umbarila, J. (2020). Estudio de habilidades metacognitivas en docentes universitarios colombianos. Revista Papeles, 12(23), 44-56.

Fecha de recibido: 10 de marzo del 2020 Fecha de aprobado: mayo 15 de 2020

* Magíster en Educación (U. Antonio Nariño, Colombia), Esp. en Docencia y Pedagogía Universitaria, Lic. en Matemáticas y Tecnología de la Información (U. La Gran Colombia) y docente de enseñanzas de las matemáticas y de la informática en colegios públicos y privados de la ciudad de Bogotá. Agradezco a la Maestría en Educación de la UAN y al equipo docente, especialmente a los profesores John Briceño y Adriana Huertas, quienes acompañaron mi proceso de formación y la realización del presente artículo. Correo electrónico: ibarrera75@uan.edu.co

*: Magíster en Evaluación y Aseguramiento de la Calidad Educativa (U. Externado de Colombia) y Lic. en Filosofía e Historia con Diplomado en Pedagogía y Docencia Universitaria (U. La Gran Colombia). Campo laboral enfocado en la gestión y enseñanzas de las ciencias sociohumanas, en cargos como docente de educación media superior, creador de contenidos pedagógicos, gestor de procesos de acreditación, evaluador de programas y políticas educativas. Coasesor de investigación externo. Correo electrónico: jose.bayona17@uan.edu.co 
instrumento y análisis de correlación entre algunas variables de caracterización de los docentes y las subcategorías del MAIT, así como correlaciones entre las mismas subcategorías. Los análisis de correlación de la muestra no evidencian correlaciones significativas entre las variables de edad, experiencia o estudios y los resultados reportados por los docentes en el MAIT. En el análisis entre subcategorías, el conocimiento declarativo y la habilidad de planeación son las subcategorías que más se relacionan con otras por su carácter fundamental. Por otra parte, el conocimiento procedimental y las habilidades de monitoreo y evaluación parecen tener poca correlación con otras subcategorías por ser las que más tienden a procesos prácticos.

Palabras clave: metacognición, MAIT, docentes universitarios, regulación, correlación.

\begin{abstract}
A study was conducted about metacognitive skills reported by 118 university teachers in Bogotá through a metacognitive awareness inventory for teachers (MAIT). Based on the data collected, a validation of the instrument and the analysis of the correlation between some characterization variables of the teachers and the MAIT subcategories are carried out, as well as correlations between the own subcategories. In the analysis of the correlation of the sample is not evident that the variables of age, experience or studies have influence on the results reported by the teachers in the MAIT. In the analysis between the subcategories, the declarative knowledge and the ability to planning are the subcategories that most relate to others because their fundamental nature. On the other hand, a procedural knowledge and monitoring and evaluation skill seems to have little correlation with other subcategories because they tend to more practical processes.
\end{abstract}

Keywords: metacognition, MAIT, University Teachers, regulation, correlation.

\title{
Introducción
}

El conocimiento de la metacognición permite el desarrollo de acciones para lograr que el estudiante (por sí solo o con el acompañamiento de un docente) evalúe y direccione su propio aprendizaje y aumente su interés en él. Para ello se deben implementar estrategias metacognitivas en el proceso de aprendizaje (tales como el autoreporte), que, teniendo en cuenta las metas propuestas, permitan enfocar conscientemente las acciones de forma estructurada hacia la resolución de un problema (Rodríguez, 1995). Huertas, Vesga y Galindo (2014) incluyen dentro de las estrategias reflexivas de las personas sobre su conocimiento, el control y la autorregulación, en coherencia con posturas como las de Flavell (1976, citado en Medel, Vilanova, Biggio, García y Martín, 2017), quien, al acuñar el término "metacognición", considera que cuando se habla de ella, necesariamente se debe hacer énfasis en el propio aprendizaje y capacidad de autoevaluación.

Al indagar sobre el proceso de aprendizaje de los estudiantes de educación universitaria, en el artículo de Campo, Escorcia, Moreno y Palacio (2016), que compara procesos de metacognición entre estudiantes colombianos y franceses, se encuentra como uno de los resultados relevantes la correlación positiva entre el nivel de rendimiento académico y los conocimientos metacognitivos, es decir, cuanto más se es consciente de las estrategias

Revista PAPELES • ISSN 0123-0670 • Vol. 12(23) • pp. 44-56 • Enero-junio de 2020 
metacognitivas, es más probable lograr mejores desempeños en el aprendizaje. Para la medición de dichas habilidades metacognitivas, Schraw y Denninson (1994), investigadores interesados en este campo, crean el inventario de conciencia metacognitiva (MAI, por sus siglas en inglés) como una herramienta que permite recoger el nivel de las habilidades de metacognición de los participantes a través de cincuenta y dos ítems (clasificados en ocho [8] subcategorías, que a su vez se encuentran divididas en otras dos categorías), que se corresponden de manera autoevaluativa con el nivel de habilidad que reconoce el participante en sí mismo para cada cuestión.

Las subcategorías del MAI recogen información sobre el conocimiento y regulación de la propia cognición, teniendo en cuenta: i) el conocimiento declarativo, ii) el conocimiento procedimental, iii) el conocimiento condicional, iv) la planificación, v) la organización, vi) el monitoreo, vii) la depuración y viii) la evaluación (Gutiérrez, Wells, Davis y Parker, 2017).

El objetivo de esta herramienta en el presente artículo es recolectar información sobre las habilidades que autorreporta una muestra de 118 docentes universitarios de 17 instituciones de educación superior (IES) de la ciudad de Bogotá (Colombia). Para realizar las mediciones sobre la autopercepción del nivel de las habilidades de metacognición en docentes universitarios se usa una versión modificada del MAI, titulado MAIT (por las siglas en inglés de la versión original del inventario de conciencia metacognitiva para docentes), traducido y obtenido en una investigación previa desarrollada por Huertas, Vesga y Galindo (2014).

A partir de la información recolectada, se realiza una validación de la versión MAIT del instrumento y se caracterizan la muestra de docentes y los niveles de conocimiento metacognitivo que autorreportan para cada una de las ocho subcategorías. Finalmente, se comparan los resultados autorreportados de las subcategorías con otros datos reportados como edad, años de experiencia docente en IES y nivel educativo de los participantes, para determinar la posible correlación existente entre estas variables y la autopercepción de las habilidades metacognitivas de los docentes participantes. Así mismo, se analizan correlaciones entre las ocho subcategorías del MAIT con el fin de vislumbrar la posibilidad de que alguna de las subcategorías implique el desarrollo de otras, o de que se encuentren relacionadas por conocimientos o habilidades similares.

\section{Marco teórico}

\section{La metacognición y sus estrategias}

Las habilidades de la metacognición, según Flavell (1979), implican la relación de tres aspectos básicos: la persona, la tarea y la estrategia. Esto supone que la metacognición es un proceso a través del cual una persona es capaz de determinar cuáles son las estrategias adecuadas para cumplir con una tarea y, en el caso educativo, lograr el aprendizaje.

Para Gutiérrez, Wells, Davis y Parker (2017), la metacognición se conoce como el proceso de reflexión sobre los propios conocimientos, así como del conocimiento sobre los propios procesos cognitivos y capacidades para regular los apropiados comportamientos frente al aprendizaje.

Debido a la importancia de las condiciones psicocognitivas para el desarrollo de las estrategias metacognitivas, estas también han sido estudiadas desde la psicología del aprendizaje. Autores como Mitchell, Busenitz, Lant, McDougall, Morse y Smith (2002) consideran que la estructura del conocimiento y las

Universidad Antonio Nariño • Facultad de Ciencias de la Educación 


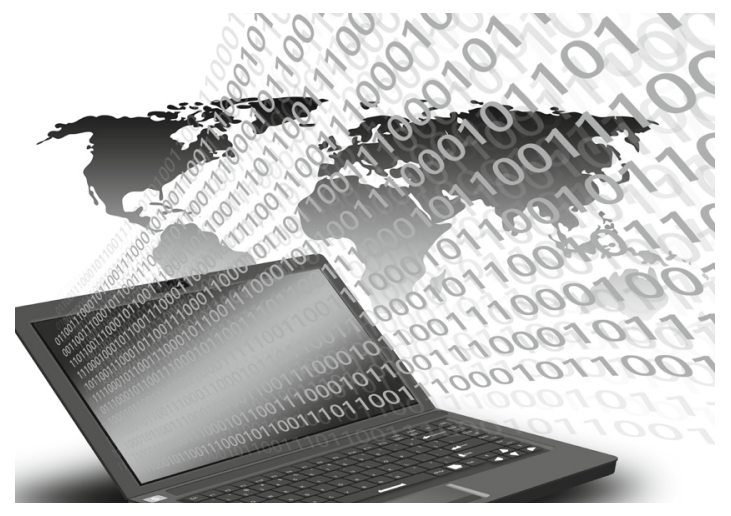

prácticas de la metacognición son un estudio que proporciona nuevos conocimientos sobre la relación entre lo psicológico y lo metodológico. Es decir, la metacognición es un puente entre el conocimiento existente y los objetivos de aprendizaje, que a través de la conciencia sobre las experiencias y la comprensión teórica o práctica de los procesos de aprendizaje debe permitirles a los sujetos tomar decisiones sobre los conocimientos y habilidades que tienen para alcanzar los objetivos de aprendizaje.

Según Gutiérrez, Wells, Davis y Parker (2017), dichos conocimientos deben enfocarse en la capacidad de dar razón sobre: i) lo que se sabe, ii) la forma en que se puede aplicar este conocimiento para solucionar una problemática y iii) el reconocimiento del contexto en que debe aplicar sus conocimientos. En cuanto a las habilidades, estas deben permitirles a las personas: iv) organizar previamente las actividades a desarrollar, v) llevar a cabo secuencialmente las actividades en pro de solucionar la problemática, vi) verificar constantemente el estado del proceso, vii) mantener, modificar o eliminar acciones y viii) determinar, finalmente, la efectividad de las acciones emprendidas frente a los objetivos propuestos. Sobre estos tres conocimientos $\mathrm{y}$ cinco habilidades se fundamentan las ocho subcategorías del instrumento usado en el presente artículo (MAI) de Schraw y Denninson (1994), las cuales implican conocimientos declarativos, procedimentales y condicionales, así como habilidades de planificación, organización, monitoreo, depuración y evaluación, para recoger información sobre la percepción que se tiene del propio conocimiento y regulación de la cognición.

\section{Métodos de enseñanza de las habilidades metacognitivas}

Es común encontrar estudiantes con grandes dificultades para comprender ciertos razonamientos, ya que ellos adoptan conductas inapropiadas en su aprendizaje, como lo es repetir manualmente las informaciones. Esta es la razón por la cual el aprendizaje debe ser más una actividad estratégica, planificada y controlada, que ayude al estudiante a comprender la importancia de los objetivos de aprendizaje y tomar conciencia del porqué de sus propios resultados de aprendizaje (Muria, 1994).

Donde son incoherentes las acciones para alcanzar un objetivo de aprendizaje con las necesidades individuales para lograrlo es donde surgen las dificultades que los estudiantes tienen para aprender significativamente, por lo cual, es fundamental que tanto el docente como el estudiante tengan la habilidad para identificar la mejor forma de enseñar o aprender (respectivamente), teniendo en cuenta las características metacognitivas que corresponden al estilo de aprendizaje del estudiante.

Según Castellá (2007), para enseñarles a los estudiantes a reconocer sus estilos de aprendizaje y a usar estratégicamente sus recursos en situaciones de aprendizaje es necesario que previamente el docente encuentre diferentes recursos y didácticas relacionadas con los contenidos curriculares, que a su vez los lleve a reflexionar sobre los conocimientos y habilidades que tienen y que necesitan alcanzar. Si el docente logra enseñar al estudiante a identificar las estrategias cognitivas más apropiadas para poder aprender según su propio estilo de 


\section{El método didáctico debe ser} metacognitivo, es decir, mediante instrucciones que le permitan a los docentes y estudiantes reflexionar por qué hacen lo que hacen, y que sean conscientes de las ventajas que tiene hacerlo de esta manera y no de otra, para que así los estudiantes se sientan motivados a aprender algo que consideran importante.

aprendizaje, logrará superar el enfoque académico superficial para, en su lugar, procurar un enfoque académico más profundo.

Inhelder y Piaget (1958) afirman que la metacognición, a partir del conocimiento reflexivo y consciente sobre lo que se piensa y conoce, puede proyectar lo que se desea conocer y las posibles formas de conocerlo. Estas actividades mentales iniciales deben permitir, principalmente: 1) establecer cuál es el objetivo de aprendizaje, 2) planificar las actividades que se consideran necesarias para lograrlo, 3) analizar el proceso de integración de los conocimientos y las habilidades que ya posee el estudiante con las actividades que se propone desarrollar para obtener nuevos conocimientos y habilidades, y 4) establecer lo que se espera lograr con cada una de las actividades a poner en marcha de acuerdo con el contexto.

En coherencia con lo anterior, Huertas, Vesga y Galindo (2014) han concluido que para definir la metacognición se requiere entender la relación que surge entre el conocimiento, las estrategias y la regulación. Para el primero de los términos (conocimiento), los autores argumentan que se refiere a las actividades metacognitivas que ayudan al reconocimiento de i) lo que se sabe (conocimiento declarativo), ii) la forma en que se puede aplicar este conocimiento para solucionar una problemática (conocimiento procedimental), iii) el reconocimiento del contexto en que se deben aplicar sus conocimientos (conocimiento condicional) y iv) la habilidad para organizar previamente las actividades a desarrollar (planificación).

Sin embargo, la enseñanza de habilidades metacognitivas no se limita a los elementos previos al proceso de aprendizaje, sino que la puesta en práctica del proceso (la didáctica del docente) es también uno de los principales medios para desarrollar la metacognición. El método didáctico debe ser metacognitivo, es decir, mediante instrucciones que le permitan a los docentes y estudiantes reflexionar por qué hacen lo que hacen, y que sean conscientes de las ventajas que tiene hacerlo de esta manera y no de otra, para que así los estudiantes se sientan motivados a aprender algo que consideran importante.

Ormrond (2005), por su parte, menciona que el aprendizaje autorregulado debe tener las siguientes fases: i) establecer objetivos, ii) planificar, iii) automotivarse, iv) controlar la atención, v) aplicar estrategias de aprendizaje, vi) autocontrolarse, vii) autoevaluarse, y viii) autorreflexionar. En esta postura se hace evidente que, aparte de las actividades previas al ejercicio metacognitivo de aprendizaje, también son fundamentales otras actividades enfocadas a regular el proceso en su desarrollo y a evaluarlo al finalizar.

Frente a dicha regulación de la cognición, las actividades que ayudan a controlar el pensamiento durante el proceso de aprendizaje son tres habilidades que permiten: i) llevar a cabo secuencialmente las actividades en pro de solucionar la problemática (organización), ii) verificar constantemente el estado del proceso (monitoreo), y iii) mantener, modificar o eliminar acciones (depuración [Huertas, Vesga y Galindo, 2014]).

Sin embargo, así como la habilidad de monitoreo hace revisiones al proceso de aprendizaje 
durante su desarrollo, se necesita de la evaluación como habilidad complementaria que verifica el nivel de alcance de los objetivos de aprendizaje al finalizar (fases o procesos completos) y hace recomendaciones para futuras estrategias (Winne y Perry, 2000). De hecho, Flavell (1976, citado en Medel, Vilanova, Biggio, García y Martín, 2017), al acuñar el término "metacognición", lo relaciona directamente al propio aprendizaje y a la capacidad de autoevaluación.

Además de los elementos metacognitivos anteriormente resaltados y enfocados a los conocimientos, estrategias y regulaciones metacognitivas de los estudiantes (relacionadas con las ocho subcategorías del MAIT), Pintrich (1999) considera que también es importante reconocer la influencia, el conocimiento y el control de los propios procesos psicoemocionales en el aprendizaje. Uno de los elementos de dicho proceso psicoemocional que más influye en el aprendizaje es el aspecto motivacional, el cual controla aspectos como: la percepción de autoeficacia, las creencias sobre la importancia de los objetivos, la orientación hacia ellos y la ansiedad ante la evaluación.

Este último aspecto metacognitivo relacionado con los factores psicoemocionales es relativamente nuevo en el estudio de la metacognición en el aprendizaje, por lo cual, no es una categoría o subcategoría del instrumento usado en este artículo (MAIT). Sin embargo, se deja planteada la posibilidad de contemplar este aspecto como un nuevo elemento para

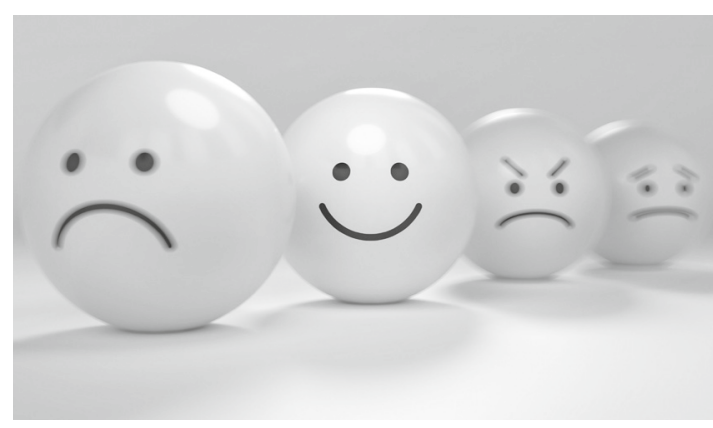

el MAIT, a partir del cual sea posible generar nuevos ítems que le den facultades al instrumento para recolectar información sobre las habilidades de manejo de las condiciones psicoemocionales y de su influencia en los procesos metacognitivos de aprendizaje.

\section{La metacognición y el docente universitario}

El desarrollo de las habilidades metacognitivas está asociado con el crecimiento intelectual, puesto que dependen de otros procesos cognitivos básicos (memoria, concentración, reflexión, autopercepción, etc.) que también se desarrollan a lo largo de la vida (Alexander, Carr y Schwanenflugel, 1995). Debido a lo anterior, uno de los propósitos que debe buscar la universidad es hacer que el estudiante tenga una evolución cognitiva enfocada hacia la consolidación de habilidades que le permitan aprender a aprender por sí mismo (Zapata, Diaz, Diaz, González y Fuentes, 2019). El fortalecimiento de estas habilidades puede ayudar a lograr un aprendizaje más exitoso, debido a que los estudiantes serán cada vez más autónomos en su aprendizaje y podrán tomar decisiones que les ayuden a profundizar teórica y prácticamente en sus condiciones metacognitivas.

Teniendo en cuenta lo anterior, el docente debe ayudar al estudiante a fortalecer el conocimiento sobre sus propios procesos mentales a través de actividades de planificación, monitoreo y evaluación que le permitan alcanzar los objetivos de aprendizaje, inicialmente guiado y posteriormente por sus propios medios.

Para Buck (2001), justamente en la transición entre el aprendizaje guiado y el aprendizaje autónomo es donde radica el gran reto del docente, debido a que su labor no solo consiste en preparar actividades de comprensión, sino también en formular estrategias que enseñen a los estudiantes a ser independientes en su proceso de aprendizaje. 
Sin embargo, el desarrollo de habilidades cognitivas no es mediado solamente por el docente, debido a que los estudiantes tienen la habilidad de crear nuevas estrategias propias del aprendizaje a partir del análisis de procesos de aprendizaje de otros. Estos procesos pueden surgir por iniciativas de los estudiantes, o guiadas por el docente que fomenta espacios de enseñanza-aprendizaje de forma grupal, en donde los estudiantes intercambian con otros (pares o docentes) experiencias y estrategias de metacognición.

Así mismo, la enseñanza de las estrategias metacognitivas no se limita a los momentos previos o paralelos al proceso de aprendizaje, sino que también se vincula con su posterior evaluación. Hacer una autoevaluación previa a los exámenes teniendo como referencia los objetivos de aprendizaje ayudará al estudiante a tener una mejor predicción sobre sus posibles resultados, lo cual, al complementarse posteriormente con una retroalimentación de los mismos, ayudará a que el estudiante aprenda sobre las estrategias y elementos que debe tener en cuenta para mejorar sus resultados en futuras evaluaciones (Saenz, Geraci, Miller y Tirso, 2017).

La importancia evidente que tiene el docente en la formación y orientación metacognitiva del estudiante se hace aún más notoria al comprender que las estrategias metacognitivas deben ser un conjunto equilibrado entre los elementos prácticos y conductuales y las operaciones de los procesos mentales, las cuales se llevan a cabo con el propósito común de mejorar el aprendizaje, es decir, la metacognición implica un saber-qué y un saber-cómo (Muria, 1994).

Por ello, el docente universitario debe ser capaz primero de comprender las implicaciones de la metacognición precisa para poder aplicarlas y direccionar a sus estudiantes hacia los objetivos de aprendizaje que les propone. El docente es, entonces, responsable primero de su propio aprendizaje, para luego poder reconocer estos mismos aprendizajes en sus estudiantes, con el fin de enseñarles desde su experiencia a alcanzarlos de la manera más eficiente (Kaplan, 2008).

Debido a lo anterior, se hace necesario evaluar en los docentes universitarios las habilidades metacognitivas que estos dominan y que, por lo tanto, pueden transmitir efectivamente a sus estudiantes e implementar en sus clases. Por ello, el presente artículo hace una primera aproximación a este objetivo por medio del MAIT, que permite recoger autopercepciones que tienen los docentes universitarios sobre sus propios conocimientos y habilidades metacognitivas, y así tener una primera visión general del nivel de desarrollo metacognitivo que los participantes de la muestra consideran tener.

\section{Metodología}

La investigación tiene un enfoque cuantitativo, debido a que se pretenden verificar las posibles relaciones entre los datos cuantificables que se obtienen de unas variables (Bayona-Umbarila, 2017).

Específicamente, para este artículo se realiza un estudio sobre las habilidades metacognitivas autorreportadas por 118 docentes universitarios de Bogotá (Colombia), pertenecientes a 17 instituciones de educación superior (IES), quienes respondieron el instrumento denominado inventario de habilidades metacognitivas para docentes (MAIT, por sus siglas en inglés), conformado por 52 ítems de escala Likert.

Los docentes participantes fueron clasificados en diferentes áreas de conocimiento: licenciados $(\mathrm{N}=42)$, ingeniería y matemáticas $(\mathrm{N}=$ 24), contabilidad, administración y economía $(\mathrm{N}=22)$; ciencias sociales $(\mathrm{N}=14)$, medios de comunicación $(\mathrm{N}=12)$, salud $(\mathrm{N}=2)$ y artes

Universidad Antonio Nariño • Facultad de Ciencias de la Educación 
$(\mathrm{N}=2)$. De ellos, $40(33,89 \%)$ eran mujeres y $78(66,10 \%)$ hombres, y sus edades oscilaban entre 25 y 77 (media: 43,9 años; desviación típica: 10,33). En cuanto a la experiencia profesional en la docencia universitaria, el valor mínimo fue de 1 año y el máximo de 50 años (media: 12,25; desviación típica: 8,23). También se preguntó a los docentes participantes por su último nivel de estudio alcanzado, que correspondía a pregrado $(\mathrm{N}=9 ; 7,6 \%)$, especialización $(\mathrm{N}=7 ; 5,9 \%)$, maestría $(\mathrm{N}=81$; $68,6 \%)$ o doctorado $(\mathrm{N}=21 ; 17,8 \%)$.

El instrumento permitió indagar sobre la autopercepción de los docentes frente a dos categorías, las cuales se dividen en ocho subcategorías relacionadas con habilidades metacognitivas.

Los docentes que respondieron de manera voluntaria el cuestionario de forma electrónica, a través de un formulario de Google, fueron contactados por correo electrónico con ayuda de las directivas de las universidades o a través de los mismos investigadores. A partir de los datos recolectados se realizó una validación del instrumento, debido a que su versión original, creada por Schraw y

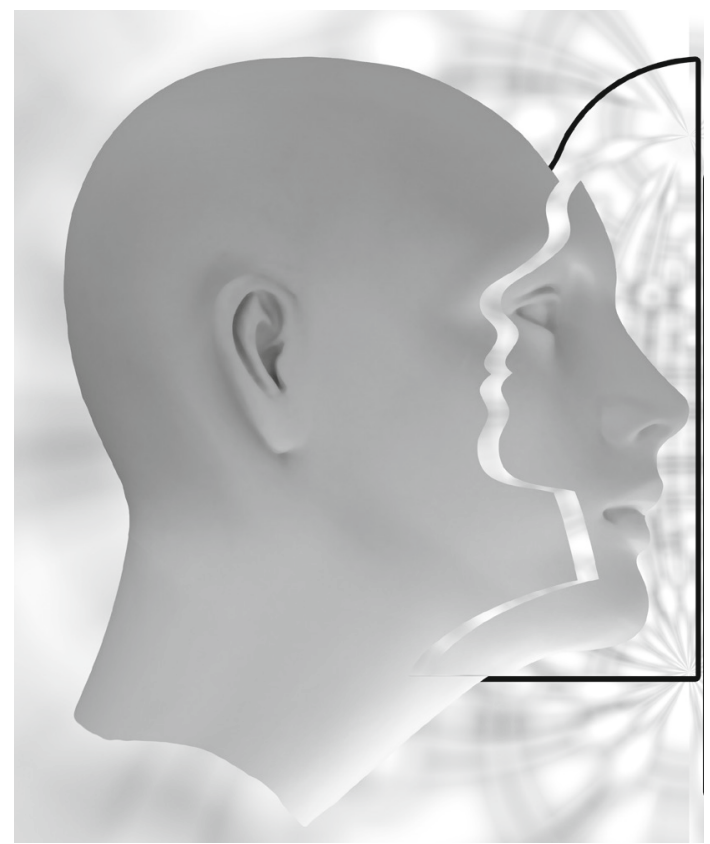

Denninson (1994), fue adaptada y traducida para aplicarla específicamente a docentes en el contexto colombiano, para posteriormente realizar estudios de correlación entre algunas variables de caracterización de los docentes y las ocho categorías del MAIT, así como correlaciones entre las mismas categorías.

\section{Resultados}

\section{Análisis de fiabilidad}

El análisis de fiabilidad del MAIT fue sistematizado a través del software Iteman 4.3, con la intención de identificar el alfa de Cronbach como indicador de la validez interna de la prueba, al determinar la efectividad de los ítems para medir la subcategoría que pretenden identificar (Lukas, 1998). Para considerar que el instrumento es fiable, es necesario que el alfa de Cronbach sea superior a 0,6, llegar a ser lo más cercano a 1,0 (sin llegar a este valor), y que la mayoría o totalidad de los ítems no afecten el nivel de confiabilidad de la prueba (Santos, 1999).
Tabla 1. Resultados análisis de fiabilidad de MAIT a través de alfa de Cronbach

\begin{tabular}{|l|c|}
\multicolumn{1}{|c|}{ Categoría } & $\begin{array}{c}\text { Alfa de } \\
\text { Cronbach }\end{array}$ \\
\hline Conocimiento declarativo & 0,898 \\
\hline Conocimiento procedimental & 0,898 \\
\hline Conocimiento condicional & 0,895 \\
\hline Planeación & 0,888 \\
\hline Organización & 0,898 \\
\hline Monitoreo & 0,889 \\
\hline Depuración & 0,901 \\
\hline Evaluación & 0,907 \\
\hline Instrumento total & 0,96 \\
\hline
\end{tabular}

Fuente: elaboración propia 


\section{Análisis de correlaciones}

Se realizaron análisis de correlaciones de Pearson bivariadas con el fin de vislumbrar la posibilidad de que alguna de las subcategorías implique el desarrollo de otras, o de que se encuentren relacionadas por conocimientos o habilidades similares. Así mismo, se realizaron correlaciones entre las subcategorías del MAIT y las variables de caracterización de los docentes para determinar la posible correlación existente entre estos elementos.

Las correlaciones se miden entre 1 y -1 , en las que los valores cercanos a 1 indican correlaciones directas muy fuertes, lo cual implica que cuando los valores de una variable aumentan, los valores de la otra variable también lo hacen, y lo mismo ocurre cuando los valores de alguna de las variables disminuyen (Bayona-Umbarila, 2017).

Por el contrario, los valores cercanos a -1 indican una correlación indirecta muy fuerte, en la cual, cuando los valores de una variable aumentan, los de la otra disminuye y viceversa. En caso de que la correlación indique un número cercano a 0 , se interpreta que la correlación entre las variables es baja o inexistente. Usualmente, para que se considere fuerte la correlación de 2 variables, es necesario que su valor sea $>0,5$ (directa) o $<-0,5$ (indirecta [Castañeda, 2010]).
Adicional al valor de la correlación de Pearson, es necesario analizar la significancia estadística de dichas correlaciones ("Sig" o "P Valor"), lo cual determina la validez de las correlaciones y confirma si estas pudieron ser o no resultado del azar o de errores en la medición (Castañeda, 2010). Para que una correlación de Pearson sea significativa, y, por lo tanto, tenga validez, el valor de significancia ("Sig" o "P Valor") debe ser $<0,05$ en un intervalo de confianza del $95 \%$, o $<0,1$ para un intervalo de confianza del 90\% (Bayona-Umbarila, 2017).

\section{Análisis de resultados}

Respecto al análisis de fiabilidad del MAIT a través del alfa de Cronbach (tabla 1), los resultados obtenidos demuestran que la adaptación del instrumento para los docentes universitarios del contexto colombiano no afectó los resultados que se han mostrado en investigaciones anteriores, donde se ha mostrado como un instrumento válido y fiable para medir la conciencia metacognitiva de los docentes en el área de la investigación educativa (Huertas, Vesga y Galindo, 2014).

En cuanto a las correlaciones entre las subcategorías de conocimientos cognitivos (tabla 2 ), todas las correlaciones son fuertes $y$ significativas.

Tabla 2. Correlaciones entre subcategorías de conocimientos cognitivos

\begin{tabular}{|c|c|c|c|c|}
\hline & & $\mathrm{CD}$ & $\mathrm{CP}$ & $\mathrm{CC}$ \\
\hline \multirow{3}{*}{ Conocimiento declarativo } & Pearson Correlación & 1 & & \\
\hline & Sig. (2-tailed) & & & \\
\hline & $\mathrm{N}$ & 118 & & \\
\hline \multirow{3}{*}{ Conocimiento procedimental } & Pearson Correlación & $.638^{* * *}$ & 1 & \\
\hline & Sig. (2-tailed) &, 000 & & \\
\hline & $\mathrm{N}$ & 118 & 118 & \\
\hline \multirow{3}{*}{ Conocimiento condicional } & Pearson Correlación & $.625^{* * *}$ & $.581^{* * *}$ & 1 \\
\hline & Sig. (2-tailed) &, 000 & ,000 & \\
\hline & $\mathrm{N}$ & 118 & 118 & 118 \\
\hline
\end{tabular}

Universidad Antonio Nariño • Facultad de Ciencias de la Educación 
Sin embargo, se destaca que en los docentes de la muestra el conocimiento declarativo tiene una muy fuerte y significativa correlación con los otros dos. Lo anterior puede deberse a que el conocimiento de aquello que se sabe es el punto de partida para determinar qué hacer con dicho conocimiento y en qué condiciones (Inhelder y Piaget, 1958). Lo anterior implica que en el proceso de la enseñanza y aprendizaje de habilidades metacognitivas es fundamental desarrollar condiciones reflexivas que permitan a los sujetos comprender a profundidad aquello que efectivamente conocen para fundamentar sobre ello la construcción de las demás estrategias.

Las correlaciones entre las subcategorías de reguladores cognitivos (tabla 3 ) también presentaron correlaciones fuertes y significativas entre todas ellas, al igual que en el análisis anterior. Entre estas correlaciones fuertes encontramos algunas con un mayor nivel de correlación, tal como lo presenta la subcategoría de planeación con las de organización y monitoreo. Lo anterior se encuentra en total coherencia con lo planteado por Schraw y Denninson (1994), quienes consideran que aquel que tiene la capacidad para proyectar las tareas y tiempos que debe emplear para alcanzar los objetivos (planeación), seguramente también se interesará por estructurar la secuencia de estas actividades (organización) y verificar durante el proceso la efectividad de las mismas para ajustarlas (monitoreo).

Así mismo, en este análisis encontramos una correlación destacada entre las subcategorías de evaluación y monitoreo, explicada posiblemente por el hecho de que la habilidad de monitoreo hace revisiones al proceso de aprendizaje durante su desarrollo y la evaluación se convierte en una habilidad complementaria que verifica el nivel de alcance de los objetivos de aprendizaje al finalizar (fases o procesos completos) y hace recomendaciones para futuras estrategias (Winne y Perry, 2000).

$\mathrm{Al}$ correlacionar las categorías de conocimientos con las de reguladores cognitivos (tabla 4), también se encontraron correlaciones

Tabla 3. Correlaciones entre subcategorías de reguladores cognitivos

\begin{tabular}{|c|c|c|c|c|c|c|}
\hline & & $\mathbf{P}$ & $\mathbf{O}$ & $\mathbf{M}$ & D & $\mathbf{E}$ \\
\hline \multirow{3}{*}{ Planeación (P) } & Pearson Correlación & 1 & & & & \\
\hline & Sig. (2-tailed) & & & & & \\
\hline & $\mathrm{N}$ & 118 & & & & \\
\hline \multirow{3}{*}{ Organización (O) } & Pearson Correlación & $.639 * \%$ & 1 & & & \\
\hline & Sig. (2-tailed) &, 000 & & & & \\
\hline & $\mathrm{N}$ & 118 & & & & \\
\hline \multirow{3}{*}{ Monitoreo (M) } & Pearson Correlación & $.733 * \%$ & $.539 \%$ & 1 & & \\
\hline & Sig. (2-tailed) &, 000 & ,000 & & & \\
\hline & $\mathrm{N}$ & 118 & 118 & 118 & & \\
\hline \multirow{3}{*}{ Depuración (D) } & Pearson Correlación & $.596^{* *}$ & $.514^{* *}$ & $.568 \%$ & 1 & \\
\hline & Sig. (2-tailed) &, 000 &, 000 &, 000 & & \\
\hline & $\mathrm{N}$ & 118 & 118 & 118 & 118 & \\
\hline \multirow{3}{*}{ Evaluación (E) } & Pearson Correlación & $.596^{* * *}$ & $.528 \%$ & $.693^{* * *}$ & $.430 * *$ & 1 \\
\hline & Sig. (2-tailed) &, 000 &, 000 &, 000 &, 000 & \\
\hline & $\mathrm{N}$ & 118 & 118 & 118 & 118 & 118 \\
\hline
\end{tabular}

Revista PAPELES • ISSN 0123-0670 • Vol. 12(23) • pp. 44-56 • Enero-junio de 2020 
fuertes y significativas para todas las parejas de variables. A pesar de lo anterior, se destacaron las correlaciones entre el conocimiento declarativo y las habilidades de organización y depuración, las correlaciones entre el conocimiento condicional y las habilidades de planeación y monitoreo, y la correlación del conocimiento procedimental con la habilidad de planeación.

Para las primeras correlaciones destacadas, sería posible afirmar que reconocer aquello que se conoce (conocimiento declarativo), puede ser un apoyo para que en dicho conocimiento se identifiquen aquellas tareas o contenidos que se dificultan (monitoreo) y, a partir de ello, se pueda estructurar una serie de acciones para solucionar dicha condición (organización [Muria, 1994]).

En cuanto a la relación entre el conocimiento condicional y las habilidades de planeación y monitoreo, estas se explican por medio de la propia definición del conocimiento condicional, el cual implica la capacidad de reconocer previamente (planeación) el cuándo y porqué de las actividades a desarrollar para lograr los objetivos a través de una verificación constante

Tabla 4. Correlaciones entre conocimientos y reguladores cognitivos

\begin{tabular}{|c|c|c|c|c|c|c|}
\hline & & $\mathrm{P}$ & $\mathrm{O}$ & M & $\mathrm{D}$ & $\mathrm{E}$ \\
\hline \multirow{3}{*}{ Conocimiento declarativo } & Pearson Correlación & $.599 \% *$ & $.657^{* * *}$ & $.579 \%$ & .610 *\% & $.429 \% *$ \\
\hline & Sig. (2-tailed) & ,000 & ,000 & ,000 & ,000 & ,000 \\
\hline & $\mathrm{N}$ & 118 & 118 & 118 & 118 & 118 \\
\hline \multirow{3}{*}{ Conocimiento procedimental } & Pearson Correlación & $.629 * *$ & $.592 \% *$ & $.559 * *$ & $.479 * \%$ & $.453^{* *}$ \\
\hline & Sig. (2-tailed) & ,000 & ,000 & ,000 & ,000 & ,000 \\
\hline & $\mathrm{N}$ & 118 & 118 & 118 & 118 & 118 \\
\hline \multirow{3}{*}{ Conocimiento condicional } & Pearson Correlación & $.647^{* * *}$ & $.513^{* * *}$ & $.642 * *$ & $.593^{* * *}$ & $.516^{* *}$ \\
\hline & Sig. (2-tailed) & ,000 & ,000 & ,000 & ,000 & ,000 \\
\hline & $\mathrm{N}$ & 118 & 118 & 118 & 118 & 118 \\
\hline
\end{tabular}

** La correlación es significativa en el nivel 0,01 (2 colas). * La correlación es significativa en el nivel 0,05 (2 colas) Fuente: elaboración propia.

Tabla 5. Correlaciones entre caracterización y subcategorías metacognitivas

\begin{tabular}{|c|c|c|c|c|c|c|c|c|c|c|c|c|}
\hline & & $\mathrm{AE}$ & $\mathrm{E}$ & $\mathrm{NE}$ & $\mathrm{CD}$ & $\mathrm{CP}$ & $\mathrm{CC}$ & $\mathrm{P}$ & $\mathrm{O}$ & M & $\mathrm{D}$ & $\mathrm{E}$ \\
\hline \multirow{3}{*}{$\begin{array}{l}\text { Años } \\
\text { Experiencia } \\
\text { (AE) }\end{array}$} & $\begin{array}{l}\text { Pearson } \\
\text { Correlación }\end{array}$ & 1 & $.663^{* *}$ & $.221 \%$ & ,139 & ,118 & ,063 & ,115 & , 170 & ,129 &,- 013 & $.191^{*}$ \\
\hline & Sig. (2-tailed) & & ,000 & 016, & 133, & 203, & 501 & 215 & 066, & 162, & 887, & ,038 \\
\hline & $\mathrm{N}$ & 118 & 118 & 118 & 118 & 118 & 118 & 118 & 118 & 118 & 118 & 118 \\
\hline \multirow{3}{*}{ Edad (E) } & $\begin{array}{l}\text { Pearson } \\
\text { Correlación }\end{array}$ & $.663^{* * *}$ & 1 &,- 049 & ,106 & ,017 &,- 069 &,- 059 & ,082 &,- 178 &,- 099 &, 050 \\
\hline & Sig. (2-tailed) & ,000 & &, 598 & ,251 & ,852 & ,455 &, 529 & ,377 &, 054 & ,288 &, 594 \\
\hline & $\mathrm{N}$ & 118 & 118 & 118 & 118 & 118 & 118 & 118 & 118 & 118 & 118 & 118 \\
\hline \multirow{3}{*}{$\begin{array}{l}\text { Nivel de } \\
\text { Estudios } \\
(\mathrm{NE})\end{array}$} & $\begin{array}{l}\text { Pearson } \\
\text { Correlación }\end{array}$ & $.221^{*}$ &,- 049 & 1 & ,029 & ,057 & , 126 & ,109 & , 166 & ,122 & ,135 & , 163 \\
\hline & Sig. (2-tailed) & ,016 &, 598 & & ,756 &, 542 & ,176 & ,239 & ,072 & ,190 & ,144 & ,078 \\
\hline & $\mathrm{N}$ & 118 & 118 & 118 & 118 & 118 & 118 & 118 & 118 & 118 & 118 & 118 \\
\hline
\end{tabular}

* La correlación es significativa en el nivel 0,01 (2 colas). * La correlación es significativa en el nivel 0,05 (2 colas). Fuente: elaboración propia. 
de la efectividad de las estrategias (monitoreo [Huertas, Vesga y Galindo, 2014]). Por otro lado, debido a que para comprender cómo se deben emplear las estrategias de aprendizaje es fundamental determinar previamente cuáles son las acciones a llevar a cabo y los tiempos de las mismas, es posible que por ello se encuentre una muy fuerte correlación entre el conocimiento procedimental y la habilidad de planeación.
Finalmente, en cuanto a las correlaciones entre las variables de caracterización (edad, años de experiencia en IES y nivel educativo) y las subcategorías del MAIT (tabla 5), el hecho de que la única correlación fuerte y significativa que se haya encontrado sea predeciblemente entre la edad y los años de experiencia, hace evidente que las variables de caracterización analizadas no son determinantes para los resultados de las subcategorías.

\section{Conclusiones}

Las correlaciones fuertes entre las diferentes subcategorías del MAIT apoyan la validación de la consistencia y fiabilidad entre sus ítems y subcategorías.

En cuanto a las correlaciones, las dos subcategorías que más correlaciones muy fuertes y significativas $(\mathrm{r}>.6, \mathrm{p}<.05, \mathrm{~N}=118)$ establecen con otras subcategorías (cuatro cada una) son las de conocimiento declarativo y planeacion. Lo anterior supone la posibilidad de que sean respectivamente el conocimiento y regulación cognitiva fundamental para el desarrollo de otras, debido a su enfoque en elementos previos (planeación) o de sustento (conocimiento declarativo).

Por su parte, el conocimiento procedimental y las habilidades de depuración y monitoreo se posicionan como las subcategorías con menos correlaciones (dos el primero y una cada una de las habilidades) muy fuertes ( $\mathrm{r}$ $>.6, \mathrm{p}<.05, \mathrm{~N}=118$ ). Esto podría implicar que, al tratarse de subcategorías relacionadas con el planteamiento de procedimientos prácticos (específicamente de evaluación para el caso de las habilidades), estas tienen dificultades para asociarse con otras subcategorías que pueden parecer inclinadas hacia procedimientos principalmente cognitivos.

La correlación entre las variables de caracterización y las subcategorías hacen evidente que (por lo menos para el caso de la muestra de este estudio) la edad, experiencia o estudios no son factores que determinen mejores o peores autopercepciones de los docentes universitarios sobre las habilidades metacognitivas.

\section{Referencias}

Alexander, J. M., Carr, M. y Schwanenflugel, P. J. (1995). Development of metacognition in gifted children: Directions for future research. Developmental review, 15(1), 1-37.

Bayona-Umbarila. J. (2017). Incidencia del uso de matrices evaluativas, en la alineación entre sus calificaciones auto, co $y$ hetero-evaluativas y en los cambios de su conocimiento sobre la utilidad de estos tipos de evaluación. (Trabajo de grado
Maestría en Evaluación y Calidad). Universidad Externado de Colombia, Bogotá, Colombia.

Buck, G. (2001). Assessing listening. Reino Unido: Cambridge University Press.

Campo, K., Escorcia, D., Moreno, M. y Palacio, J. (2016). Metacognición, escritura y rendimiento académico en universitarios de Colombia y Francia. Avances en Psicología Latinoamericana, 34(2), 233-252. 
Castañeda, M. B. (2010). Procesamiento de datos y análisis estadísticos utilizando SPSS: un libro práctico para investigadores y administradores educativos. EDIPUCRS.

Castellá, J. M. (2007). Entenderse en clase; estrategias discursivas $y$ habilidades comunicativas. España: Graó.

Flavell, J. (1979). Metacognition and cognitive monitoring- A new area of cognitive-developmental inquiry. American Psichology, 34, 906-911.

Flavell, J. (1976). Metacognitive Aspects of Problem Solving: The Nature of Intelligence. Hillsdale: N. J. Erlbaum.

Gutiérrez de Blume, A. P., Wells, P., Davis, C. A. y Parker, J. (2017). "You Can Sort of Feel It": Exploring Metacognition and the Feeling of Knowing Among Undergraduate Students. The Qualitative Report, 22(7).

Huertas Bustos, A. P., Vesga Bravo, G. J. y Galindo León, M. (2014). Validación del instrumento "Inventario de habilidades metacognitivas (MAI)" con estudiantes colombianos. Praxis \& Saber, 5(10), 56-74.

Inhelder, B. y Piaget, J. (1958). The growth of logical thinking from childhood to adolescence: An essay on the construction of formal operational structures. Psychology Press, (22).

Kaplan, A. (2008). Clarifying Metacognition, Self-Regulation, and Self-Regulated Learning: What's the Purpose? Educational Psychology Review, 20(4), 477-484.

Lukas, J. F. (1998). Análisis de items y de tests con ITEMAN. Leioa, España: Universidad del País Vasco.

Medel, G. A., Vilanova, S. L., Biggio, C., García, M. B. y Martín, S. S. (2017). Estrategias metacognitivas y concepciones sobre el aprendizaje en la formación inicial de profesores universitarios del área de ciencias exactas y naturales. Informes Psicológicos, 17(1), 35-51.

Mitchell, R. K., Busenitz, L., Lant, T., McDougall, P. P., Morse, E. A. y Smith, J. B. (2002). Toward a theory of entrepreneurial cognition: Rethinking the people side of entrepreneurship research. Entrepreneurship theory and practice, 27(2), 93-104.

Muria Vila, I. (1994). La enseñanza de las estrategias de aprendizaje y las habilidades metacognitivas. Perfiles educativos, (65).

Pintrich, P. R. (1999). The role of motivation in promoting and sustaining self-regulated learning. International journal of educational research, 31(6), 459-470.

Rodríguez, M. E. (1995). "Hablar" en la escuela: ¿Para qué? ... ¿Cómo? Lectura y Vida. Revista Latinoamericana de Lectura, 16(3), 31-40.

Saenz, G. D., Geraci, L., Miller, T. M. y Tirso, R. (2017). Metacognition in the classroom: The association between students' exam predictions and their desired grades. Consciousness and cognition, (51), 125-139.

Santos, R. (1999). Cronbach's alpha: A tool for assessing the reliability of scales. Journal of extension, 37(2), 1-5.

Schraw, G. y Dennison, R.S. (1994). Evaluación de la conciencia metacognitiva. Psicología educativa contemporánea, 19 (4), 460-475.

Winne, P. H. y Perry, N. E. (2000). Measuring self-regulated learning. In M. Boekaerts, M. Zeidner y P. R. Pintrich (Eds.), Handbook of self-regulation (pp. 531-566). San Diego, EU.: Academic.

Zapata, N. A., Diaz, M. A. A., Diaz, H. H. A., González, R. y Fuentes, A. R. (2019). Estrategias metacognitivas y comprensión lectora en estudiantes universitarios. Apuntes Universitarios, 9(1), 36-45. 